\title{
The Evolution of Best-in-Class Pharmacy Management Techniques
}

\author{
Developing integrated data systems and involving pharmacists as \\ integral members of a comprehensive health care team will help \\ managed care organizations maximize patient care and control costs.
}

\section{Beth Boland}

\section{Aenser}

BETH BOLAND, M.S., is a Senior Consultant with Blue Cross and Blue Shield Association, Chicago, IL.

ACKNOWLEDGEMENT: The author wishes to acknowledge the following individuals for their contributions in developing this article: Rob Seidman, Blue Cross of California; Annette Boyer, Highmark Blue Cross Blue Shield; and Grant Lawless, M.D., Highmark Blue Cross Blue Shield.

Copyright (O 1998, Academy of Managed Care Pharmacy, Inc. All rights reserved.
$\mathrm{N}$ umerous Blue Cross and Blue Shield plan administrators have strategized about the future direction of their pharmacy programs, expressing concerns about controlling rising pharmacy costs. Their concerns spurred a national study of best practices in Health maintenance organization (HMO) pharmacy program management, and the results were intriguing.

\section{INDUSTRY TRENDS}

The health care industry is becoming increasingly integrated and regional in focus as the market responds to consumer and governmental pressure to continually. reduce the cost of care. In an effort to drive administrative economies of scale, payors and providers-and even distributors and manufacturers - of health care services are consolidating to gain efficiencies, reduce costs, and enhance their access to capital (see Figure 1).

The consolidation trend among health plans hasn't been limited to publicly traded, for-profit players. The Blue Cross and Blue Shield (BCBS) system also has shown evidence of substantial consolidation, resulting in a decrease in the total number of BCBS plans from 86 plans in 1985 to the 56 plans licensed in 1998. Nearly a third of these recent mergers were transactions that crossed state boundaries.

In addition to efforts to gain administrative efficiencies from consolidation, health plans also are attempting to control medical costs by working more closely with network providers. Most health plans have developed innovative programs to align the financial incentives of physicians more closely with those of the health plan, primarily through risk-sharing programs and distributions of bonus payments for achieving specific goals.

While some plans have had success in controlling administrative costs through consolidation and medical costs through developing risk-sharing arrangements with providers, they have had only limited success in controlling pharmacy costs. HMOs across the industry are experiencing increases in prescription drug claims costs in the neighborhood of $10 \%-20 \%$ annually, a rate that often has exceeded the predictions of both management and Wall Street financial analysts.

In the first quarter of 1997, Foundation Health Systems experienced a $1.6 \%$ increase in its medical loss ratio, twothirds of which was attributed to increases in pharmacy costs. Also in 1997 , the management at Independent Health, western New York's largest HMO, claimed that escalating drug costs caused by a $5 \%$ year-to-year increase in the use of prescriptions, combined with an $8 \%$ average increase in the cost of prescriptions, required an immediate management response. Independent Health raised premiums by $8.9 \%$ for members with a prescription drug rider and instituted a $50 \%$ coinsurance for injectable drugs. Other health plans, including BCBS plans, also have reported escalating pharmacy costs that often were significantly higher than projections.

Across the country, health plans are challenged to stem this rate of increase as the FDA approval process becomes more streamlined and unprecedented 
Figure 1. Recent Consolidation and Nationalization

\begin{tabular}{l} 
Payors \\
\hline Aetna/U.S. Healthcare \\
United/MetraHealth \\
Cigna/Healthsource \\
Foundation/HSI \\
Pacificare/FHP
\end{tabular}

Providers

Columbia/HCA

Phycor/NAMM

MedPartners/ Mullikin/ Caremark

Distributors
Rite Aid/Thrifty Payless
CVS/Revco/Big B
JC Penney (Thrift Drugs)/Eckerd

Manufacturers

Upjohn/Pharmacia AB

Glaxo/Burroughs Wellcome

Hoechst/Marion Merrill Dow

numbers of new pharmaceuticals are released each year. Many of these new therapies are new molecular entities or high-priced, one-of-a-kind drugs that are likely to find their way onto drug formularies of most HMOs without a waiting period. Furthermore, guidelines proposed by the FDA to tighten drugswitching practices used by pharmacy benefit management (PBM) companies and health plans may, if approved, further impede an HMO's ability to effectively control pharmacy costs at the point of purchase.

To help BCBS plans respond to the challenges presented by these rising pharmacy costs, Strategic Consulting Services, a division of the Blue Cross and Blue Shield Association, conducted a study to answer several key questions, including:

$\Delta$ How are today's HMOs responding to these increasing pharmacy costs?

$\Delta$ How will the leading HMOs of tomorrow respond, and how will their capabilities improve over time?

To respond to these questions, we conducted interviews with several industry experts, assessed existing research and literature, and spoke with the management of several BCBS plans. Key findings included:

A Most of today's HMOs are adopting piecemeal approaches to controlling costs.

A Leading HMOs are adopting incentive-based approaches to cost control.

A Successful HMOs of the future will develop a fully integrated approach to patient care.

\section{THE PIECEMEAL APPROACH}

Most HMOs today are adopting discrete sets of initiatives designed to curb pharmacy cost increases, rather than implementing integrated prescription drug management strategies. Such HMOs are focusing mainly on three key areas for cost containment: tighter control of the pharmacy benefit; increased costsharing with members; and physician education programs.

\section{Control of the Pharmacy Benefit}

In recent years, design of the pharmacy benefit has evolved to reflect the market's changing financial structure. Modifying the design of the pharmacy benefit is perhaps one of the most straightforward opportunities to influence pharmacy costs. Today, most health plans use some type of drug formulary to reduce costs by promoting the use of preferred drugs. However, not all plans manage the drug formulary aggressively (i.e., use either a closed drug formulary or an open drug formulary with a three-tier copay structure) or aggressively require preauthorization of certain drug claims.

A three-tier copay structure typically features three copayment levels (e.g., $\$ 5 / \$ 10 / \$ 15)$ to differentiate between on-formulary generic, on-formulary brand, and off-formulary prescriptions, respectively. Utilization of either a closed drug formulary or a three-tier copay is critical to ensuring drug formulary compliance by members and physicians. The use of a tightly controlled formulary typically allows a plan to drive additional volume to key drugs and negotiate more favorable volume contracts with those drug vendors, reducing overall pharmacy costs.

Many HMOs that are focusing on pharmacy cost control are moving decisively toward designing pharmacy benefit packages with partially closed or closed drug formularies. Most plans that stop short of full drug formulary closure tend to utilize and enforce a preauthorization requirement for coverage of nonformulary treatments.

In addition to tighter drug formulary management, HMOs also are increasing their use of mandatory generic substitution and therapeutic interchange as levers to help them control escalating drug costs at the point of purchase. Still other HMOs are attempting to gain greater discounts with drug dispensers by negotiating exclusive contracts with selected large, national pharmacy networks and mail-order suppliers.

Blue Cross of California (BCC) offers several HMO products with mandatory generic substitution and a closed drug formulary. In products with a closed drug formulary, 18 drugs are available as therapeutic equivalents to formulary drugs, which can be prescribed if medically necessary. These drugs represent $30 \%$ of drug costs but are prescribed for only $2 \%$ of members. Use of the therapeutic equivalents has saved $\mathrm{BCC}$ about $4 \%$ in pharmacy costs. The preferred drug list minimizes any negative impact on members while maximizing cost savings.

\section{Member Cost-Sharing}

Many HMOs are further altering the pharmacy benefit by imposing additional cost-sharing mechanisms on members, hoping to induce more efficient utilization of prescription drugs. Not all plans offer coverage of the pharmacy benefit. Many plans that do offer coverage as standard or as a rider have instituted a two-tier copay or coinsurance system to influence members to use generic drugs when available. Health plans that have moved toward tighter drug formulary management are 
Continued from page 367

offering products with a three-tier copay. Plans trying to aggressively control pharmacy costs are starting to increase the amount of the copay or coinsurance, particularly for high-cost, high-frequency drugs, such as patient self-injectables.

These initiatives begin to more closely align the financial incentives of the member with those of the health plan to reduce total prescription drug costs.

\section{Physician Education Programs}

Recognizing that the prescribing physician has a tremendous impact on pharmacy costs, many insurers have developed programs to educate physicians about the financial impact of their prescribing behavior. These programs, which typically stop short of imposing financial incentives, often use a combination of physician report cards and peer review methods to educate physicians.

Physician report cards provide physicians with a graphic or tabular report of their prescription utilization as it compares to peers in the same specialty and geographic area. These reports communicate key indicators on each physician's utilization and cost by drug category, including cost per prescription, drug formulary usage rate, and generic substitution rate. The data from these reports often are incorporated into a peer review process during which physician outliers are identified and educated about proper prescribing techniques.
These programs represent a good start in helping plans develop the tools and expertise necessary to promote effective physician risk-sharing and incentive programs. However, by themselves, these education programs often fail to make physicians accountable for the results of their performance, resulting in minimal cost savings.

\section{THE INCENTIVE-BASED APPROACH}

Health plans with the greatest success in managing prescription drug costs have developed approaches that align the financial incentives of key parties, including those of physicians and PBM companies, with those of the plans.

\section{Physician Incentive Programs}

Successful physician incentive programs build on the foundation laid by physician education initiatives by imposing meaningful financial rewards and, potentially, penalties to influence physician prescribing behavior (see Figure 2). These programs can use either a utilization-based approach or a cost-based approach. Utilization-based programs reward physicians for meeting or exceeding specific utilization targets, including the number of prescriptions per member per month (PMPM), the percentage of generic utilization, and the percentage of drug formulary utilization.

Figure 2. Critical Success Factors for Physician Incentive Plans

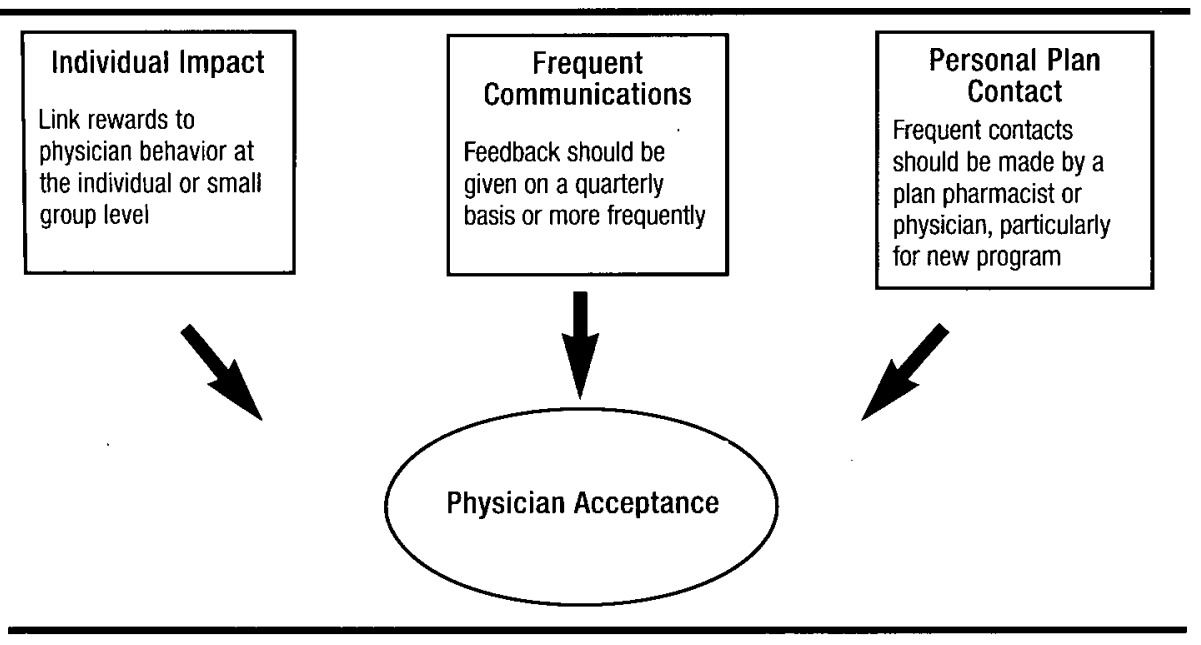

Cost-based programs, in contrast, pay physicians on costs PMPM rather than on specific utilization parameters. Payments to physicians can be based on pharmacy costs only or on total medical costs.

Regardless of the type of program developed, plans that use pharmacybased physician incentive programs should be sensitive to the potential for backlash from consumers, who may fear that HMOs will withhold coverage of necessary drugs as a result.

Highmark Blue Cross Blue Shield in Pennsylvania has developed a physician incentive program to compensate physicians via a variable bonus amount based on total medical cost targets and specific quality measures. A physician receives a higher potential bonus payment if total medical costs are below targets, and a lower payment if above. Bonus payments also depend on the physician meeting several utilization and quality standards. This program provides physicians the information they require to be successful and allows physicians to make their own decisions about medical and pharmacy cost trade-offs.

While conclusive financial evidence on these types of programs is not yet available, preliminary results involving smaller groups of physicians and their enrollees show moderate cost declines.

\section{PBM Incentive Programs}

Many HMOs outsource a variety of functions to PBMs, particularly those functions that benefit from economies of scale and are not key competencies upon which plans place a high strategic value. For those plans that want to cultivate successful, long-term relationships with PBMs, two key areas of focus emerge: aligned incentives, and the ability to access and receive integrated data.

Some health plans own a PBM, which may give them an initial advantage in terms of aligned incentives over plans with contractual relationships. However, plans with contractual PBM relationships can develop incentivebased contracts to successfully achieve the same objectives. These contracts can 
Continued from page 370

include built-in performance guarantees, including claim adjudication accuracy, transaction processing times, system availability goals, dispensing rate and accuracy, concurrent/retrospective drug utilization review (DUR) edits, and pharmacy network distribution parameters. If the PBM does not meet the targets, it is assessed financial penalties. By ensuring an adequate level of performance on key quality parameters, HMOs can eliminate the expense required to research and correct potentially costly errors.

In addition, some HMOs are utilizing PBMs for more advanced functions, which are typically clinical in nature. These functions are relatively new to the PBM industry and may represent the greatest opportunity for PBMs to help health plans successfully manage pharmacy benefit cost and utilization. These advanced functions may include prospective DUR, case management, disease state management, physician interventions, and outcomes research. Each of these functions requires the PBM to work closely with the health plan on a customized basis. As these functions mature, it will become increasingly critical that the PBM and health plan work together to accommodate the growing expectations for accountability and quantifiable, demonstrable results.

The ability to access and receive timely pharmacy data from the PBM, and to integrate this pharmacy data with plan pharmacy and medical data, also is becoming increasingly important. Integrated medical and pharmacy data are critical to the development of disease management programs and to preparing for the successful model of the future, as described later.

\section{Carve-Out/Disease Management Programs}

Disease management seems to be near the top of most plans' to-do lists, although very few plans have welldeveloped, integrated programs in place today. Most plans consider the ability to integrate medical and pharmacy data to be critical to the development of successful disease management programs. This capability would allow plans to quantify the impact of specific drug interventions on other medical costssuch as emergency room visits, physician office visits, and hospitalizationsfor broad patient populations. Without integrated, online databases, this process is patient-specific, manual, and timeintensive, making it nearly impossible to track outcomes from specific drug interventions administered for specific diagnoses on a broad, system-wide basis.

While some plans are developing pharmacy-based disease management programs, many plans prefer to wait until they can effectively integrate medical and pharmacy data before developing comprehensive programs. To date,

Figure 2. Fully Integrated Approach to Patient Care

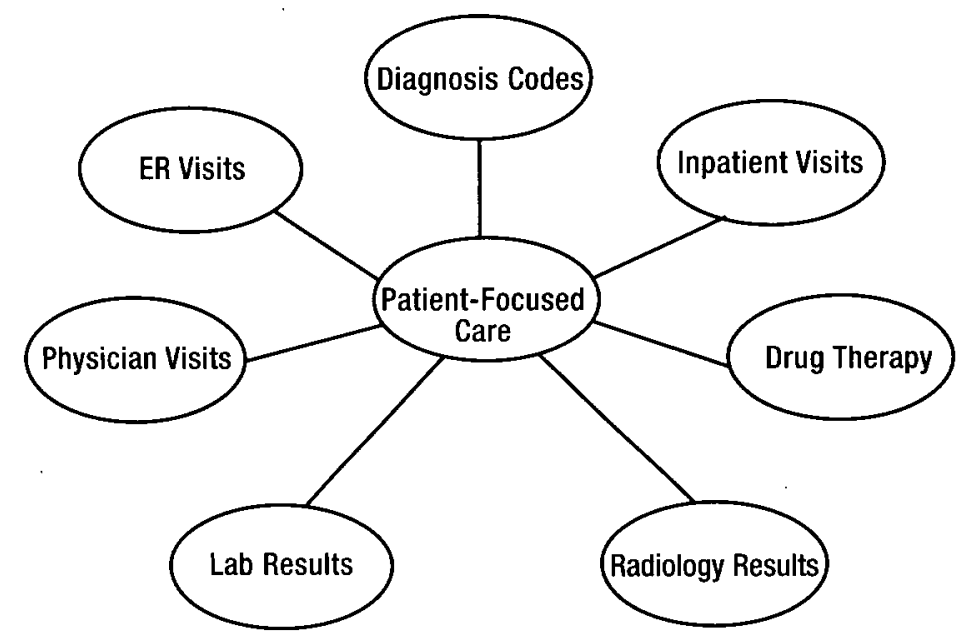

most plan efforts in this area have focused on satisfying National Committee for Quality Assurance and Healthcare Employer Data and Information Set guidelines. More innovative plans have begun to integrate medical and pharmacy data for specific patient subgroups and to develop programs that identify specific high-risk individuals and proactively intervene to prevent negative outcomes.

Plans that are developing disease management programs are focusing their efforts on high-impact, chronic conditions such as asthma, diabetes, cancer, hypertension, and coronary heart disease. Once affected members have been identified, the plan works with physicians to apply treatment protocols that most effectively manage the care. More sophisticated programs are tracking and reporting outcomes to report on quality indicators and refine treatment protocols.

Blue Cross of California (BCC) and Wellpoint Pharmacy Management, its PBM, share ownership. Through this close relationship, the plan can integrate claims and prescription data to more easily identify and manage patients with specific diagnoses. BCC can create disease profiles for members with selected disease states and can monitor medication compliance. Providers receive information on the patient's condition that encourages compliance, suggests drug therapies, and provides cost management information. For example, BCC's asthma pharmacotherapy optimization program focuses on identifying asthmatics undergoing regular beta agonist treatment in the absence of an antiinflammatory agent. The plan also has developed Members-at-Risk and Seniorsat-Risk programs that notify primary care physicians of members with a high potential for negative drug interactions.

\section{THE INTEGRATED APPROACH}

Forward-thinking HMOs are building on the capabilities acquired through their disease management programs and are preparing themselves to develop a fully integrated approach to patient care. 
Rather than focusing on selected chronic diseases and managing components of care, these plans are adopting an approach that considers not just members with identified disease states, but all members. An integrated approach will enable plans to more effectively determine the impact of drug and other therapies on patient outcomes, rather than simply assessing the costs associated with the therapies themselves. Therefore, an integrated process will improve the overall allocation of health care resources in the long run by enabling better medical decisions (see Figure 2).

Plans adopting this long-term approach are seeking to manage patient care that includes:

$\Delta$ promoting wellness;

A identifying population risk factors;

A diagnosing specific conditions;

A applying integrated treatment protocols (with drug therapy, if applicable)

$\Delta$ tracking, measuring, and reporting outcomes; and
$\boldsymbol{\Delta}$ updating protocols as necessary.

To effectively execute the above tasks, plans must have the capability to integrate, manage, and utilize data from multiple sources. Successful plans of the future are working toward this goal and are beginning to put the pieces together.

\section{CONCLUSION}

While piecemeal and incentive-based approaches to pharmacy cost management are prevalent and meet with moderate success, these approaches are inherently limited by their sole focus on pharmacy costs. For most plans, the ability to manage the entire medical dollar, control overall medical costs, and effectively allocate health care resources are the ultimate goals. These goals are most effectively achieved by promoting wellness and early detection and by aggressively managing illness--the main goals of an integrated approach to patient care.

Plans that want to move to an inte- grated approach to patient care can begin by ensuring that all plan components and processes target integration, communication, and data access. Plan reporting structures and staffing should allow for interaction and communication between pharmacy and other medical/clinical staff and should move away from viewing pharmacy as an area separate and distinct from medical management. A pharmacist should be included as an integral member of the health care decision-making team assembled at health plans. Medical management and other clinical programs should focus on effectively integrating drug therapies and other treatments and on identifying conclusions, dependencies, and relationships

between therapies and outcomes. Most important, HMOs should develop integrated data capabilities that allow timely access to data from disparate sources to build the foundation for a fully integrated program.

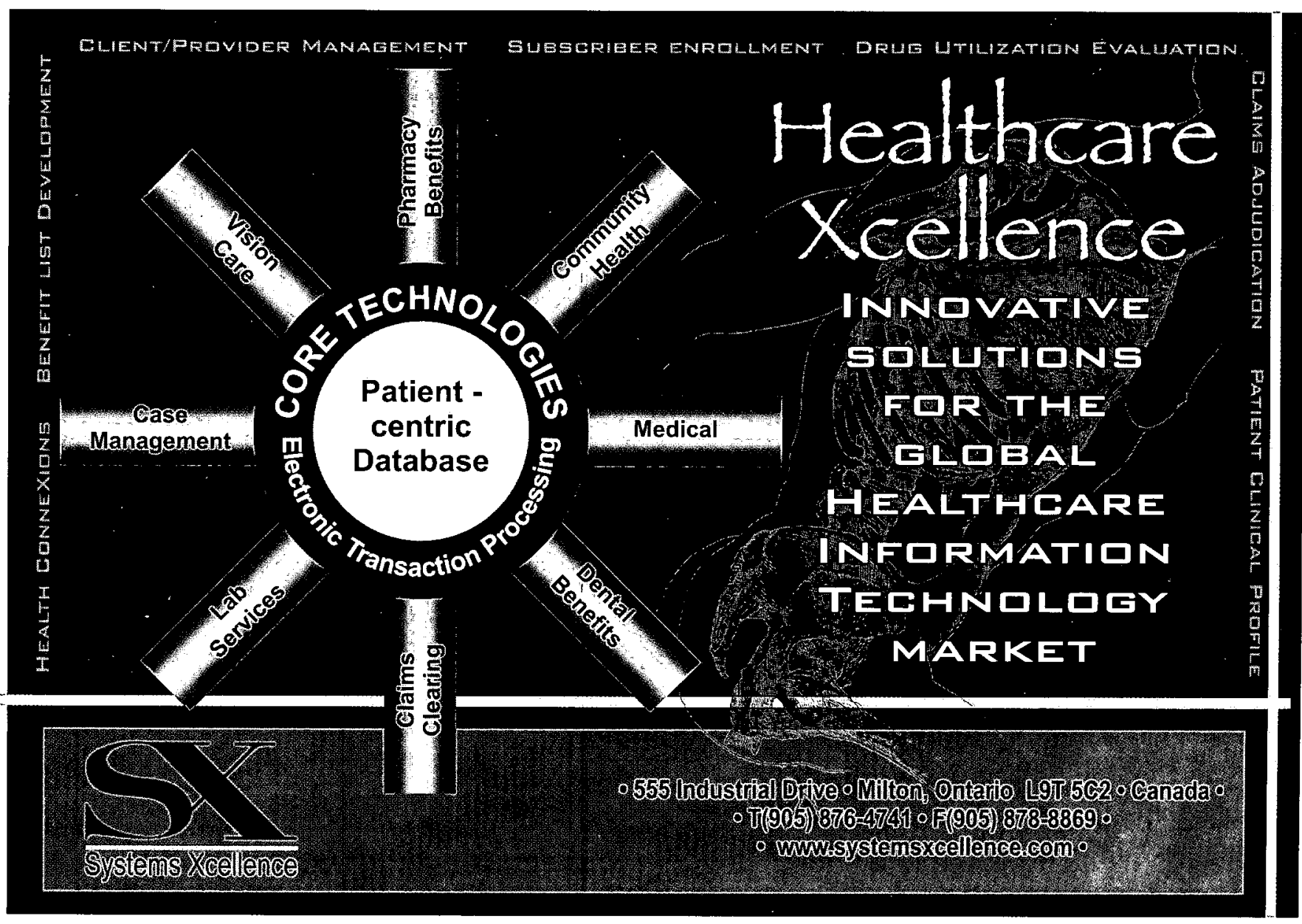

